\title{
Erythema induratum (of Bazin) in a patient with endometrial tuberculosis
}

\author{
H.J. De Silva, A.K.E. Goonetilleke, N.R. De Silva, L.R. Amarasekera ${ }^{1}$ and \\ U.S. Jayawickrama
}

General Hospital, Colombo 8, Sri Lanka and ${ }^{1}$ Faculty of Medicine, Kynsey Road, Colombo 8, Sri Lanka.

\begin{abstract}
Summary: We report erythema induratum in a patient who was found to have active endometrial tuberculosis. This case report emphasizes the importance of an exhaustive search for active tuberculosis in patients with erythema induratum, especially in countries where tuberculosis is prevalent, as the indiscriminate treatment of erythema induratum with steroids may be harmful.
\end{abstract}

\section{Introduction}

Erythema induratum is a chronic, recurring, nodular and ulcerative disorder of the lower legs which is predominantly seen in women. ${ }^{1,2,3,4}$ Because mycobacteria cannot be recovered from the lesion ${ }^{3,5,6}$ a tuberculous aetiology has been questioned. It is however thought that a tuberculous delayed hypersensitivity reaction involving the blood vessels of the legs may be responsible for the lesion, ${ }^{4,5}$ the evidence for this being that mycobacterial antigens have been found in them. ${ }^{3}$ Active tuberculosis (TB) elsewhere in the body, such as in the lungs, pleura, pericardium, peritoneum and cervical lymph nodes, ${ }^{2,5}$ is found only rarely in these patients ${ }^{6}$ and most authors therefore accept two types of erythema induratum one with and one without tuberculous causation. ${ }^{3,6}$ The term erythema induratum of Bazin is usually reserved for those cases with tuberculous causation. ${ }^{6,7,8}$

\section{Case report}

A 36 year old woman was admitted to our unit complaining of fever and nodular swellings of her legs of seven days duration. The nodules, which had developed over a course of two days, were present mainly on the calves, and were painful. She had noticed that the skin over the lesions was darkened. There was no ulceration. The fever had been mild and mainly in the evenings and was associated with night sweats.

Correspondence: H.J. De Silva, M.B., B.S., M.D., Gastroenterology Unit, The Radcliffe Infirmary, Oxford OX2 6HE.

Accepted: 29 September 1987
She had been married for 19 years but had no children. She had attained menarche at the age of 12 years and the menstrual periods were regular until shortly before her marriage. They then became irregular, sometimes occurring about once in every two or three months. The menstrual flow lasted from 5 to 7 days. No clots were passed. A dilatation and curettage of her uterus had been performed 11 years ago. The curettings had not been sent for histology. Her menstrual cycle was still irregular at the time of her present admission to hospital. She had no anorexia or loss of weight and a review of her other systems drew no further complaints.

Inquiry into her past history revealed that 16 years ago she had been successfully treated for a left basal pneumonia. Records showed that it had responded to a 10-day course of intramuscular penicillin. Sputum examined for acid-fast bacilli (AFB) at that time was negative. She had no past history, family history or contact history of TB.

On examination she was of average build. She was afebrile (and remained so for the duration of the hospital stay) and had no mucosal pallor, icterus or clubbing. She had subcutaneous nodules in both calves and on the heel of her left foot. They were about 1 to $3 \mathrm{~cm}$ in diameter, slightly tender and attached to the overlying skin which was purplish in colour. There was no ulceration and she had no inguinal lymphadenopathy. The rest of her skin, nails and hair were normal. Examination of her cardiovascular and respiratory systems, abdomen, nervous system and joints revealed no abnormality. Internal examination (both rectal and vaginal) was also normal.

(C) The Fellowship of Postgraduate Medicine, 1988 
Although the nodular skin lesions were characteristic of erythema induratum, erythema nodosum was considered in the differential diagnosis.

\section{Investigations}

The erythrocyte sedimentation rate (ESR) was $130 \mathrm{~mm}$ in the first hour. Routine haematology and biochemical tests were within normal limits. The rheumatoid factor (latex flocculation) and antinuclear factor titres were negative, the serum albumin $29 \mathrm{~g} / 1$ and globulins $34 \mathrm{~g} / \mathrm{l}$. The antistreptolysin ' $O$ ' titre was 100 Todd units. A Mantoux test using $0.1 \mathrm{ml}$ of one in 10,000 dilution, purified protein derivative was strongly positive (induration of $35 \mathrm{~mm}$ diameter with vesiculation).

Six days after admission, a biopsy of a subcutaneous nodule (13 days old), showed a normal epidermis with the subcutaneous fat having a large number of follicles composed of epithelioid cells, mononuclear cells and multinucleated giant cells of Langhans surrounding areas of caseation necrosis. Vasculitis was also marked. The appearances were characteristic of erythema induratum. Culture of the lesions did not yield mycobacteria.

Following the histology report a search was made for an active focus of TB. Chest X-ray showed a mild honeycombed appearance in a small area of the left lung base suggestive of bronchiectasis. Sputum for acid-fast bacilli was negative on five occasions. A closed liver biopsy showed only reactive inflammatory changes in the portal tracts. There were no microgranulomata. A dilatation and curettage of the uterus was then performed. The histology revealed a large number of granulomata composed of epithelioid cells, multi-nucleated giant cells of Langhans and mononuclear cells. This was strongly suggestive of endometrial TB. This diagnosis was confirmed 6 weeks later by the positive culture of Mycobacterium tuberculosis from the curettings.

After the endometrial histology report, the patient was started on rifampicin, isoniazid, ethambutol and pyridoxine. When reviewed 20 days later the subcutaneous lesions were not palpable and the skin discolouration had faded to a light brownish colour. When reviewed again after 5 months of treatment there was no evidence of the skin lesion, the ESR was $11 \mathrm{~mm}$ in the first hour and according to the patient her menstrual cycles occurred regularly once in about every 30 days.

\section{Discussion}

As mentioned earlier the term erythema induratum of Bazin is usually reserved for cases of erythema induratum with a tuberculous causation. ${ }^{6,7,8}$ As far as we are aware there have been no previous reports of an association between erythema induratum and endometrial TB. However, this association is not unexpected. The diagnosis of active TB in patients with erythema induratum may sometimes be difficult as the skin lesions themselves do not yield mycobacteria ${ }^{3,5,6}$ and a positive tuberculin test is not conclusive evidence of TB in these patients. ${ }^{3,4}$ Furthermore the TB focus may be in an unusual or relatively inaccessible site or, as is the case with endometrial TB, the disease may remain silent for long periods of time, ${ }^{9}$ requiring an exhaustive search.

There have been reports of erythema induratum not responding to anti-TB drugs but responding well to corticosteroids. ${ }^{5,10}$ These were presumably cases of erythema induratum without a tuberculous causation. However, we wish to emphasize that, especially in countries where TB is prevalent, even if an intensive search for active TB fails, it may be safer for patients with erythema induratum to be first treated with a full anti-TB regime. A good response to these drugs has the added value of being used as a diagnostic test. ${ }^{11}$ Resolution of the lesions may be slow and anti-TB drugs should be continued for at least a year. ${ }^{1}$ If there is no response to these drugs, there may be a place for corticosteroids, which may then be used relatively safely, without the risk of dissemination from an undiagnosed TB focus.

\section{References}

1. Rook, A. \& Williamson, D.S. In: Textbook of Dermatology. Blackwell, Oxford, 1979, pp 694-695.

2. Sneddon, I.B. \& Church, R.E. In: Practical Dermatology. Edward Arnold, London, 1983, pp 131132.

3. Fitzpatrick, T.B., Eisen, A.Z., Wolff, K., Freedberg, I.M. \& Austen, K.F. In: Dermatology in General Medicine. McGraw-Hill, New York, 1979, pp 14851487.
4. Symmers, W. St. C. In: Systemic Pathology, Vol. 6. Churchill Livingstone, London, 1980, pp 2742-2743.

5. Forstrom, L. \& Winklemann, R.K. Acute panniculitis. Arch Dermatol 1977, 113: 909-917.

6. Pierini, L.E., Abulafia, J. \& Wainfeld, S. Idiopathic lipogranulomatous hypodermitis. Arch Dermatol 1968, 98: 290-298. 
7. Montgomery, H., O'Leary, P.A. \& Barker, N.W. Nodular vascular diseases of the legs. JAMA 1945, 128: $335-340$.

8. Michelson, H.E. Inflammatory nodular lesions of the lower leg. Archives of Dermatology and Syphilology 1952, 66: 327-331.

9. Dewhurst, J. In: Integrated Obstetrics and Gynaecology for Post-graduates. Blackwell, Oxford, 1981, pp 532-535.
10. Van der Lugt, L. Some remarks about tuberculosis of the skin and tuberculids. Dermatologica 1965, 131: 266-272.

11. Winkinson, D.S. The vascular basis of some nodular eruptions of the legs. $\mathrm{Br} J$ Dermatol 1954, 66: 201213. 